\title{
Manejo de la cesación tabáquica entre residentes de cardiología de Iberoamérica
}

\author{
Smoking cessation management among lberoamerica's cardiology residents
}

\begin{abstract}
Sebastián García-Zamora1* , Augusto J. Lépori', Antonio Jordán², Yalile Nauhm³, Romina Roif*, Gabriel Paredes ${ }^{5}$, Alan Sigal', Marcos Ferrández-Escarabajal ${ }^{6}$, Laura Pulido ${ }^{1}$ y Jesús Álvarez-García ${ }^{7}$

${ }^{1}$ Consejo Argentino de Residentes de Cardiología (CONAREC), Buenos Aires, Argentina; ${ }^{2}$ Asociación de Fellows y Residentes Cardiólogos de México (ARCAME), Ciudad de México, México; ${ }^{3}$ Sociedad Chilena de Cardiología y Cirugía Cardiovascular (SOCHICAR), Santiago de Chile, Chile; ${ }^{4}$ Comité de Cardiólogos en Formación de Uruguay (CCFUR), Montevideo, Uruguay; ${ }^{5}$ Instituto Nacional de Cardiología Prof. Dr. Juan A. Cattoni, Asunción, Paraguay; ${ }^{6}$ Servicio de Cardiología del Hospital Clínico San Carlos, Madrid, España; ${ }^{7}$ Servicio de Cardiología del Hospital de la Santa Creu i Sant Pau, Instituto de Investigación Biomédica Sant Pau, CIBERCV, Universidad Autónoma de Barcelona, España
\end{abstract}

\begin{abstract}
Resumen
Introducción y objetivos: Si bien los cardiólogos asisten cotidianamente a pacientes que sufren daño por el tabaquismo, no se conoce el grado de formación que reciben sobre esta problemática durante su residencia. Debido a ello nos propusimos evaluar las preferencias y prácticas de los residentes de cardiología para la cesación tabáquica de los pacientes que asisten. Materiales y métodos: Encuesta cerrada, prefijada, voluntaria y anónima entre médicos que realizaban la especialidad de cardiología en cinco países de Latinoamérica y España. Resultados: Se encuestaron 716 residentes: un 62.4\% de Argentina, un $19 \%$ de México, un $6.8 \%$ de España, un 6.7\% de Chile, un $3.2 \%$ de Uruguay y un $1.9 \%$ de Paraguay. Con respecto a la importancia que asignaban a esta problemática (empleando una escala de 1-10), el 85.8\% le asignó a esta pregunta una puntuación de 8 o mayor. Mientras el $80.5 \%$ de los participantes expresó dar consejo breve antitabáquico sistemáticamente, solamente un $27.7 \%$ empleaban terapia farmacológica con este fin. Entre quienes no empleaban terapia farmacológica, el 58.3\% manifestó que el motivo era no encontrarse familiarizados con los tratamientos. El $62.9 \%$ de los encuestados dijo no haber recibido ningún tipo de formación en esta problemática. Aquellos residentes que recibieron algún tipo de formación manifestaron sentirse más preparados $(p<0.0001)$. Conclusión: Encontramos un bajo conocimiento sobre el tratamiento farmacológico y relativamente poca seguridad por parte de los residentes de cardiología para brindar asistencia en cesación tabáquica. Consideramos esencial incluir este tópico en la formación de los futuros cardiólogos a fin de lograr una prevención cardiovascular más integral.
\end{abstract}

Palabras clave: Cesación tabáquica. Nicotina. Vareniclina. Bupropión. Formación. Residentes cardiología.

\begin{abstract}
Introduction and objectives: Although cardiologists frequently assist patients who suffer damage from smoking, the degree of training they receive to manage this problem during their residency is unknown. Because of this, we'd proposed to evaluate the preferences and practices of cardiology residents for smoking cessation of the attending patients.
\end{abstract}

Correspondencia:

*Sebastián García-Zamora

E-mail: sebagz83@gmail.com
Fecha de recepción: 12-08-2020

Fecha de aceptación: 20-11-2020

DOI: $10.24875 /$ ACM.20000381
Disponible en internet: 03-05-2021 Arch Cardiol Mex. 2021;91(4):431-438 www.archivoscardiologia.com 1405-9940 / (c) 2020 Instituto Nacional de Cardiología Ignacio Chávez. Publicado por Permanyer. Este es un artículo open access bajo la licencia CC BY-NC-ND (http://creativecommons.org/licenses/by-nc-nd/4.0/). 
Materials and methods: Closed, prefixed, voluntary and anonymous survey among doctors who carried out the specialty of cardiology in 5 countries of Latin America and Spain. Results: 716 residents were surveyed; 62.4\% from Argentina, 19\% from Mexico, 6.8\% from Spain, 6.7\% from Chile, 3.2\% from Uruguay, and 1.9\% from Paraguay. When asked about the importance they assigned to this problem (using a scale of 1-10), $85.8 \%$ assigned this question a score of 8 or higher. While $80.5 \%$ of the participants expressed giving short anti-tobacco advice routinely, only $27.7 \%$ used pharmacological therapy for this purpose. Among those who did not use pharmacological therapy, $58.3 \%$ said that the reason was not being familiar with the treatments; $62.9 \%$ of the surveyed said they had not received any type of training in this problem. Those residents who received some type of training reported feeling more prepared for this $(p<0.0001)$. Conclusion: We found that cardiology residents have a low knowledge of pharmacological treatment and relatively low confidence to provide assistance in smoking cessation. This topic should be included in the training of future cardiologists in order to achieve a more comprehensive cardiovascular prevention.

Key words: Smoking cessation. Nicotine. Varenicline. Bupropion. Training. Residents. Cardiology.

\section{Introducción}

El tabaquismo constituye la principal causa de muerte en países desarrollados ${ }^{1,2}$ y en muchos en vías de desarrollo. Su prevalencia en Latinoamérica y España es elevada, ubicándose, con ligeras variaciones, entre el 20 y el $33 \% \%^{3-8}$.

La Organización Mundial de la Salud ha desarrollado un proyecto denominado « 25 por 25 », con el objetivo de reducir para el año 2025 un 25\% las muertes por las cuatro enfermedades crónicas no transmisibles más frecuentes (enfermedades cardiovasculares, pulmonares, diabetes y cáncer) ${ }^{9}$. Siendo el tabaquismo un factor de riesgo prevenible y modificable con repercusión directa en al menos tres de los cuatro ejes de esta iniciativa, la lucha contra esta adicción resulta central ${ }^{10}$.

Los pacientes que fuman y sufren eventos cardiovasculares se encuentran más receptivos a recibir intervenciones antitabaco ${ }^{11}$, y los cardiólogos asistimos continuamente a individuos que sufren perjuicios por esta adicción ${ }^{1}$. Por ello, nuestras acciones resultan claves para aconsejar y acompañar a los fumadores en el proceso del cese tabáquico ${ }^{12}$, práctica recomendada en la atención de nuestros pacientes, siendo incluso un marcador de calidad ${ }^{13,14}$. Sin embargo, y paradójicamente, el abordaje de esta patología no se encuentra específicamente contemplado en los programas de formación de la especialidad.

Por lo tanto, nuestro objetivo es evaluar el grado de conocimiento, preferencias y prácticas de los residentes de cardiología de diferentes países de Latinoamérica y España respecto a las herramientas para el abandono del tabaquismo en los pacientes hospitalizados por un evento cardiovascular.

\section{Métodos}

\section{Diseño del estudio}

Entre los meses de noviembre de 2018 y octubre de 2019 se realizó un estudio transversal mediante una encuesta cerrada, prefijada, voluntaria y anónima entre residentes y jefes de residentes de cardiología pertenecientes a centros públicos y privados de Argentina, Chile, España, México, Paraguay y Uruguay. Para ello se diseñó un cuestionario específico con 29 preguntas, 17 obligatorias y 12 condicionadas, mediante la aplicación Google Forms. La encuesta fue diseñada íntegramente por los autores del presente estudio, y se distribuyó por medio de los comités de residentes de las sociedades nacionales de cardiología de cada país mediante correo electrónico y/o mensajes de texto, invitando a participar a los potenciales encuestados por medio de un muestreo no probabilístico por conveniencia.

La encuesta incluyó un preámbulo en el que se especificaba que formaba parte de un proyecto de investigación, los objetivos de este y su carácter voluntario. Asimismo, constaba el tratamiento anónimo de los datos y que la información recogida estaría protegida de acuerdo con las leyes de protección de datos vigentes en cada país. Con ese fin, y para evitar un condicionamiento en las respuestas, se omitieron datos de filiación como la edad, el sexo, centro de formación, año de formación y ciudad de residencia. Finalmente, se indicaba que el encuestado aceptaba de forma voluntaria su participación, otorgando su consentimiento de forma tácita al responder la encuesta.

Para las preguntas en las cuales se solicitó a los participantes que indicasen la importancia que asignaban a un determinado tópico se empleó una escala 
lineal entre 1 y 10, implicando una puntuación más alto mayor importancia.

No se contó con financiamiento ni becas de estímulo de organismos públicos ni privados de ningún país para diagramar, llevar adelante ni publicar el presente estudio.

\section{Análisis estadístico}

Las variables continuas se expresaron, según su distribución, como media y desviación estándar o mediana y rango intercuartílico. La normalidad de la distribución se evaluó mediante la comparación de la relación entre media y mediana, asimetría y curtosis, herramientas gráficas (histogramas, gráfico de probabilidad normal, etc.) y el test de Shapiro-Wilk. Las variables categóricas se expresaron como porcentajes y se analizaron por el método de chi cuadrado o mediante el test exacto de Fisher, dependiendo de la frecuencia relativa de los valores esperados. Las variables numéricas se analizaron con el test $t$ de Student $\mathrm{o}$ con el método de suma de rangos de Wilcoxon, de acuerdo con la presencia o no de una distribución gaussiana respectivamente. Se valoró el grado de asociación entre las variables mediante el coeficiente de correlación de Spearman. En todos los casos se asumió un error alfa del $5 \%$ para establecer la significación estadística. Los análisis fueron realizados con STATA versión 13.0.

\section{Resultados}

\section{Características demográficas de los participantes}

Respondieron la encuesta 716 residentes. El $62.4 \%$ realizaba su formación en Argentina, el 19\% en México, el $6.8 \%$ en España, el 6.7\% en Chile, el $3.2 \%$ en Uruguay y el $1.9 \%$ en Paraguay. El $56.3 \%$ de los participantes pertenecían a centros públicos. Un $13.4 \%$ de los residentes encuestados fumaban y un $15.4 \%$ manifestaron ser exfumadores. Se observó una tendencia hacia una mayor tasa de tabaquismo entre residentes de México (19.1\%) y Argentina (13.2\%), mientras que la mayor proporción de exfumadores se encontró entre los residentes de Chile (25\%) y Uruguay (17.4\%) $(p=0.07)$. Ningún participante de Paraguay manifestó haber fumado nunca (Fig. 1).

Al preguntar respecto a la importancia que asignaban a las intervenciones para el cese tabáquico durante la hospitalización, el $85.8 \%$ de los participantes respondió con una puntuación igual o superior a 8 , mientras que solo un $4.2 \%$ la puntuaron con un 5 o menos.

\section{Consejería en cesación tabáquica}

El $80.5 \%$ de los residentes manifestó que aconsejaba "sistemáticamente» a sus pacientes fumadores que abandonaran esta adicción, el 15.2\% expresó hacerlo «a menudo", mientras que el restante $4.3 \%$ expresó no hacerlo «nunca o solo esporádicamente». Respecto al grado de adicción a fumar, el $41.9 \%$ de los participantes manifestó que preguntaba "sistemáticamente» a sus pacientes sobre esto, un $26 \%$ expresó que lo hacía «frecuentemente», un $20.5 \%$ admitió hacerlo solo "ocasionalmente» y un $11.6 \%$ contestó que «nunca» evaluaba este aspecto, siendo estas diferencias estadísticamente significativas según el país de residencia $(p<0.001)$ (Tabla 1).

De los participantes que evaluaban el grado de adicción al menos ocasionalmente $(n=633)$, solo el $9 \%$ refirió utilizar un cuestionario validado, siendo el test de Fagerström el más empleado (40.4\%). El 53.4\% manifestó que evaluaba la adicción de manera subjetiva durante la anamnesis, el $20.5 \%$ expresó que utilizaba el autorreporte de adicción por parte del paciente y el $17.1 \%$ refirió no contar con un método específico para ello.

El $37.4 \%$ de los encuestados refirió contar en su centro con especialistas en cesación tabáquica y un 22.9\% manifestó que tenían guías o protocolos para evaluar pacientes fumadores ingresados en sus servicios.

Respecto a las estrategias concretas para la cesación tabáquica, un $41.5 \%$ de los residentes empleaban estrategias no farmacológicas además del consejo médico. En este subgrupo $(n=297)$ la intervención más empleada fue la derivación de los pacientes a especialistas (en su centro o en otro), bien como única estrategia $(53.9 \%)$ o junto a otras herramientas como material informativo en papel o audiovisual sumado a la derivación (20.5\%).

\section{Tratamiento farmacológico}

Al preguntar respecto a la prescripción de fármacos para favorecer el cese tabáquico, el $27.7 \%$ respondió que los empleaba al menos "ocasionalmente». Entre estos participantes ( $n=198)$, el $54.6 \%$ utilizaban solamente un fármaco: el $30.8 \%$ alguna forma de terapia de reemplazo nicotínico (TRN), el 12.6\% bupropión, el 


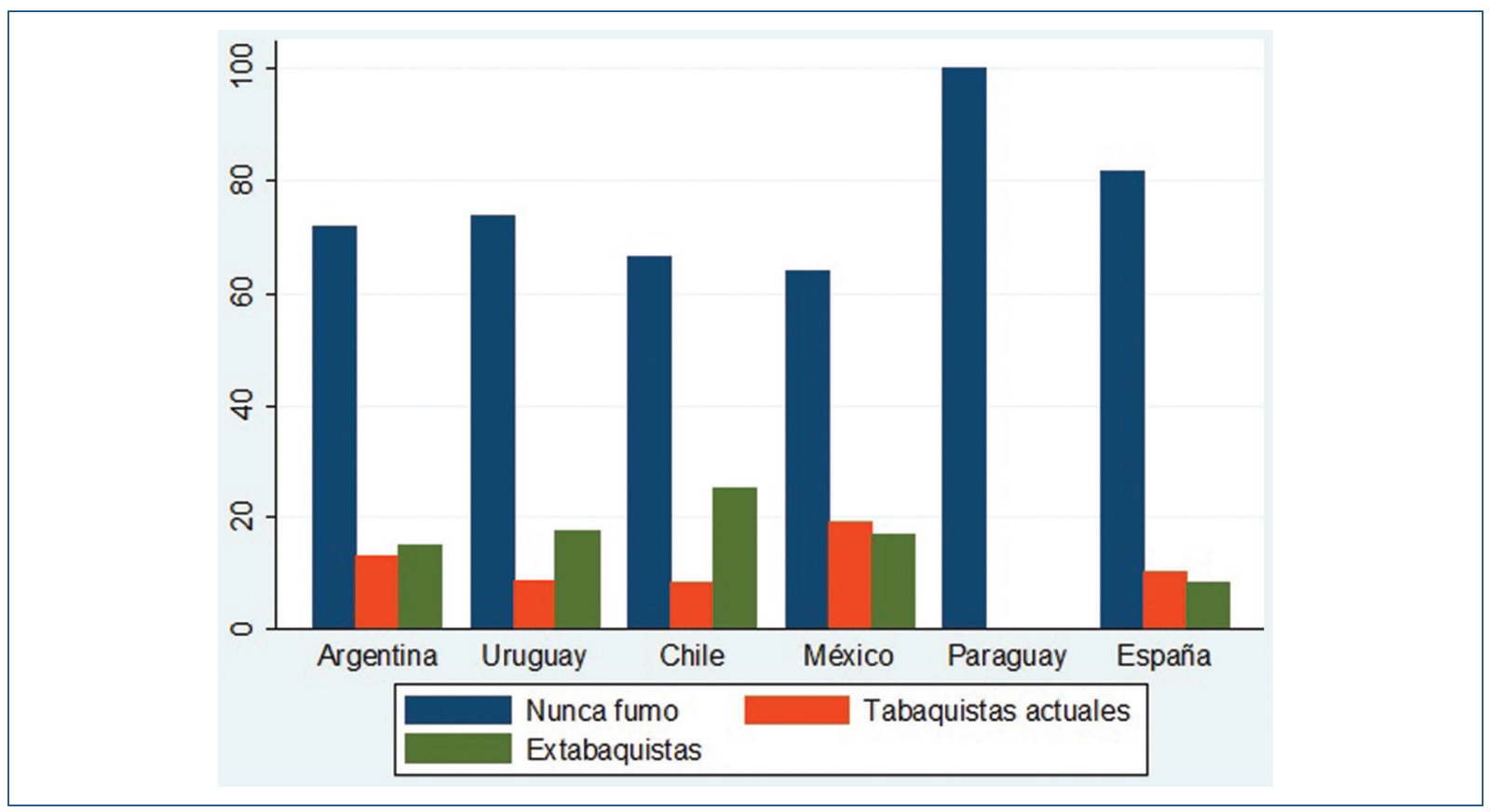

Figura 1. Adicción al tabaco según país de residencia. Se aprecia una tendencia no significativa hacia diferencias en el grado de adicción al tabaco de acuerdo con el país de residencia ( $p=0.07)$.

Tabla 1. Evaluación del grado de adicción de los pacientes al tabaco según país de residencia. Se observan importantes diferencias respecto del reporte de la evaluación del grado de adicción según país de residencia $(p<0.0001)$

\begin{tabular}{|l|c|c|c|c|}
\hline País & Nunca & Ocasionalmente & Frecuentemente & Siempre \\
\hline Argentina & $11 \%$ & $22.6 \%$ & $27.7 \%$ & $38.7 \%$ \\
\hline Chile & $6.3 \%$ & $22.9 \%$ & $27.1 \%$ & $43.7 \%$ \\
\hline España & $20.4 \%$ & $22.5 \%$ & $40.8 \%$ & $16.3 \%$ \\
\hline México & $8.8 \%$ & $8.1 \%$ & $16.9 \%$ & $66.2 \%$ \\
\hline Paraguay & $15.3 \%$ & $46.2 \%$ & $15.4 \%$ & $23.1 \%$ \\
\hline Uruguay & $30.4 \%$ & $30.4 \%$ & $17.4 \%$ & $21.8 \%$ \\
\hline Total & $11.6 \%$ & $20.5 \%$ & $26 \%$ & $41.9 \%$ \\
\hline
\end{tabular}

$7.6 \%$ benzodiazepinas y el $3.5 \%$ vareniclina. El $22.8 \%$ estaban familiarizados con el uso de dos fármacos (mayoritariamente TRN junto a otra alternativa), mientras que los restantes $22.7 \%$ refirieron emplear tres 0 cuatro fármacos dentro de sus alternativas terapéuticas. Al preguntar en qué momento de la hospitalización de un paciente fumador le ofrecían terapia farmacológica para abandonar su adicción, el 20.4\% manifestó que lo hacían desde la unidad de cuidados críticos, el $30.6 \%$ lo comenzaba cuando la persona se encontraba en sala de hospitalización, mientras que el $49 \%$ restante lo iniciaba en el momento del alta hospitalaria o en la consulta ambulatoria.

Entre los participantes que no empleaban ninguna terapia farmacológica en sus pacientes fumadores ( $n=518$ ), el $58.3 \%$ expresó que el motivo era no encontrarse familiarizados con estos fármacos, seguido de la falta de disponibilidad en la farmacia de su institución, o el elevado coste de estos (24.5\%). Entre otras causas menos frecuentes, se encontró temor a aumentar los eventos cardiovasculares (3.5\%), temor a los efectos adversos en general (4.1\%) o ausencia de capacidad para su prescripción (2.7\%). 


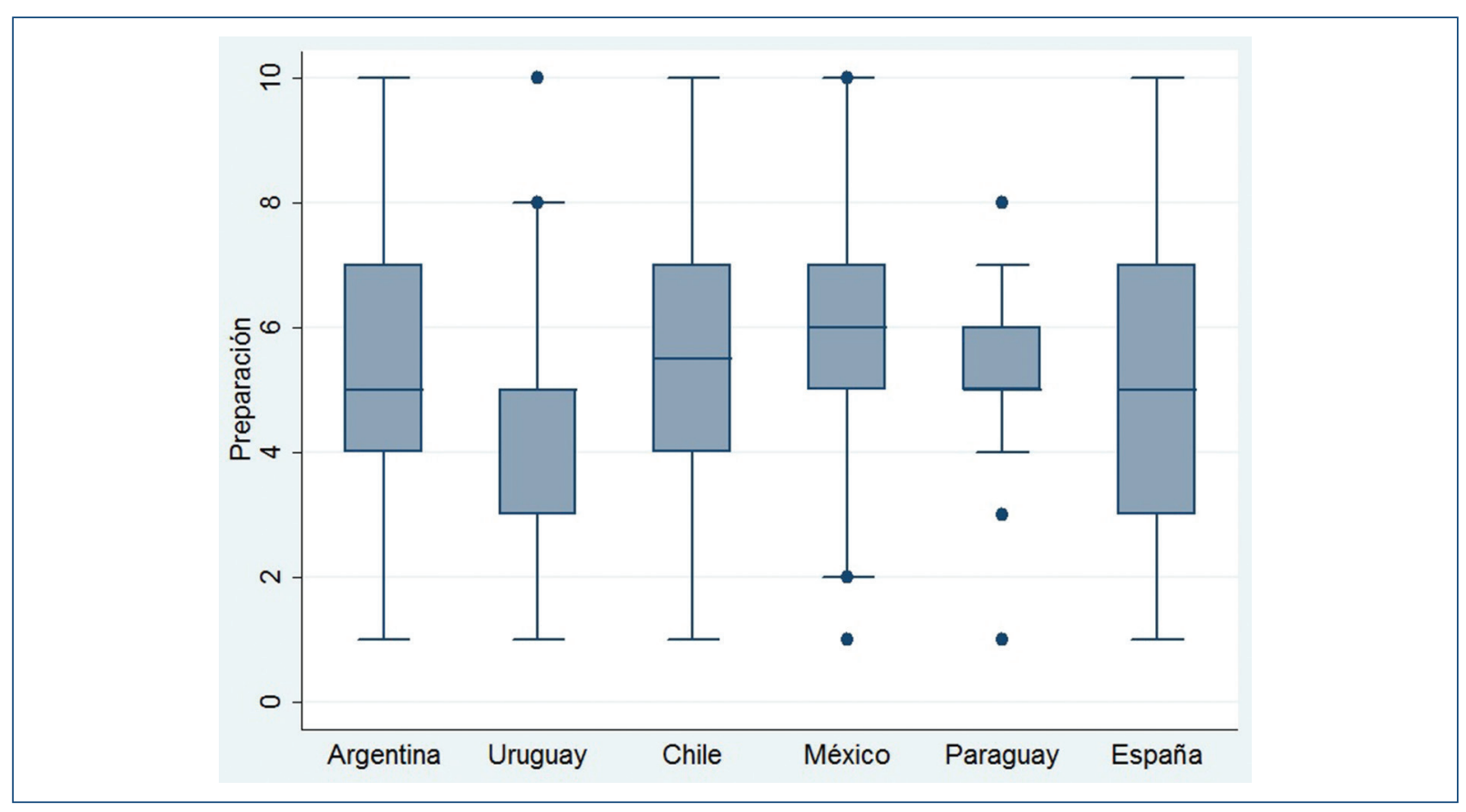

Figura 2. Autorreporte del grado de preparación para asistir a pacientes fumadores a que abandonen su adicción, según país de residencia. Se observa la diferencia de las medianas de puntuación de los residentes de acuerdo con el país de residencia ( $p=0.03$ ). Argentina 5 (RIC 4-7); Uruguay 5 (RIC 3-5); Chile 5.5 (RIC 4-7); México 6 (RIC 5-7); Paraguay 5 (RIC 5-6); España 5 (RIC 3-7). RIC: rango intercuartiles.

\section{Formación en tabaquismo y consideraciones finales}

Respecto a la formación en cesación tabáquica, el $62.9 \%$ expresó no haber recibido ningún tipo de instrucción al respecto. Entre aquellos que habían recibido algún grado de formación $(n=262)$, el $73.2 \%$ habían tenido clases sobre el tema, un $26 \%$ sesiones o revisiones bibliográficas y un $0.8 \%$ cursos específicos en la materia. Al solicitar a los encuestados que expresaran su grado de preparación para abordar pacientes con esta problemática, la media de respuestas fue de $5.4 \pm 2.2$. Aquellos participantes que recibieron algún tipo de formación en cesación tabáquica manifestaron sentirse más preparados $(6.2 \pm 1.9$ vs. $4.9 \pm 2.2$; $p<0.0001$ ), aunque solo un $15.6 \%$ de los encuestados respondió a esta pregunta con una puntuación igual a 8 o superior. Los residentes de México y Chile manifestaron estar ligeramente más preparados $(p=0.03)$ (Fig. 2).

Respecto de la relevancia de esta temática para su práctica cotidiana, el $88 \%$ de los participantes le asignó una puntuación igual o mayor a 8 y un $56.4 \%$ puntuó esta pregunta con 10. No encontramos diferencias en las respuestas entre quienes habían recibido formación en cesación tabáquica y quienes no $(9.0 \pm 1.5$ vs.
$9.1 \pm 1 ; p=0.28)$, ni tampoco de acuerdo con el país de residencia (Fig. 3). Finalmente, al explorar el grado de correlación entre la relevancia asignada a la cesación tabáquica y la autopercepción respecto al grado de preparación personal para afrontar el problema encontramos una muy débil correlación entre ambas $(r=0.26)$.

\section{Discusión}

Nuestro estudio entre residentes de cardiología de cinco países de Latinoamérica y España demuestra que a pesar de que el tabaquismo es un problema relevante y reconocido, es muchas veces soslayado durante la formación de estos. De esta forma, un elevado número de residentes manifestó no sentirse capacitados para asistir a sus pacientes en el cese de esta adicción, especialmente en lo que respecta a prescripción de fármacos con probada eficacia para este fin $^{13}$.

La residencia es un momento crítico en el proceso de formación de los médicos, y por ende propicio para el entrenamiento en cese tabáquico. Si bien múltiples estudios han evaluado la factibilidad de entrenar a residentes para abordar esta problemática ${ }^{12,15-17}$, se desconoce el grado de preparación adquirido en 


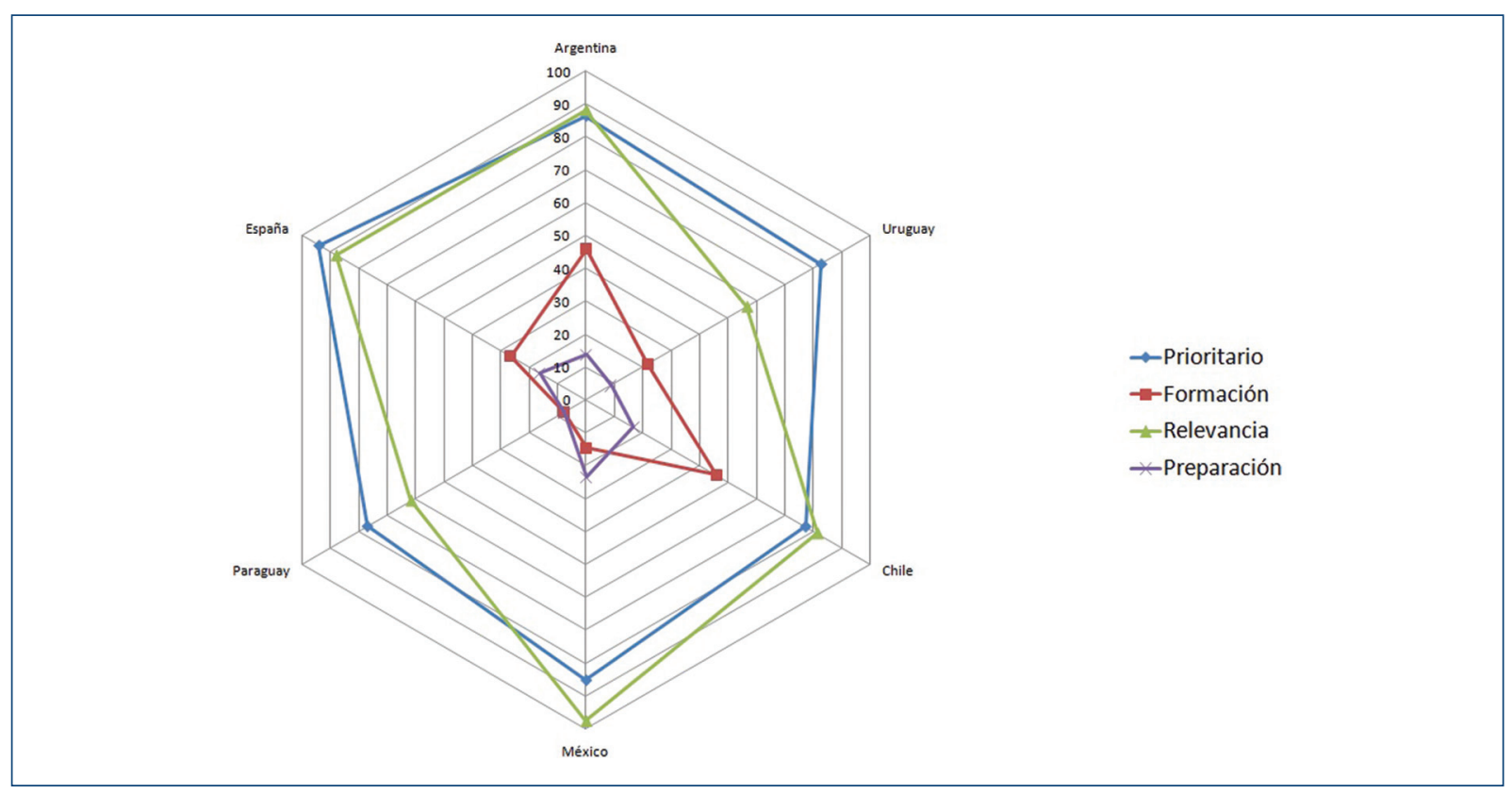

Figura 3. Valoración de los participantes según país de residencia, respecto a la relevancia, prioridad, grado de formación y seguridad para la atención de pacientes con adicción al tabaquismo. Expresado en porcentaje de participantes que valoraron estos ítems con 8 o más puntos. La mayoría de los residentes consideró la cesación tabáquica un tema relevante y prioritario en la atención de pacientes fumadores hospitalizados. Si bien se aprecian diferencias en el autorreporte del grado de formación sobre la temática, un bajo porcentaje de residentes de todos los países expresó sentirse muy preparados para esto.

nuestros programas de formación en cardiología. Una encuesta realizada en EE.UU. evaluó programas de centros formadores en cardiología, revelando que únicamente el $24 \%$ de estos incluían una rotación de un mes para sus residentes en un departamento de prevención cardiovascular. De aquellos que rotaban en estas áreas, solamente el 5\% tenían contacto con algún programa de cesación tabáquica ${ }^{18}$. Fuera de este ámbito, algunos trabajos han explorado, de manera similar a nuestro estudio, el grado de preparación para la ayuda en el cese tabáquico. Así, un estudio entre 292 residentes de oftalmología de hospitales de EE.UU. concluyó que si bien el $78 \%$ de los participantes preguntaba siempre o frecuentemente a sus pacientes si fumaban, solamente un $51 \%$ dialogaba con ellos sobre sus deseos de abandonar esta práctica ${ }^{19}$. Otro estudio reciente evaluó residencias de ginecología y obstetricia. Los autores encontraron que el $60 \%$ de los centros no incluían estos tópicos en sus programas de formación, mientras que aproximadamente la mitad de quienes lo hacían, destinaban menos de una hora al año para la educación en esta área ${ }^{20}$.
A pesar del conocimiento del daño derivado del tabaquismo, muchos fumadores no cesarán su adicción hasta que presenten complicaciones relacionadas a esta ${ }^{1}$. De hecho, el tabaquismo continúa siendo uno de los principales factores de riesgo relacionado con causas de muerte modificables en todo el mundo ${ }^{21}$. Tanto es así, que el cese tabáquico es la principal medida tanto en prevención cardiovascular primaria como secundaria ${ }^{22}$.

Por otra parte, si bien los pacientes hospitalizados dejan de fumar voluntaria o involuntariamente (debido a políticas de hospitales libres de humo de tabaco), la mayoría vuelve a hacerlo después del alta ${ }^{23,24}$. Esto constituye una verdadera oportunidad perdida, ya que múltiples trabajos han demostrado que las personas que son hospitalizadas por un problema vinculado a la adicción del tabaco se encuentran más receptivas a la hora de recibir consejos para cesar su adicción ${ }^{25,26}$. Además, las personas que reciben consejos del personal de salud para dejar de fumar logran hacerlo más frecuentemente ${ }^{25-28}$. Sin embargo, y paradójicamente, un número importante de personas fumadoras no reciben intervenciones para cesar su 
práctica, a pesar de estar en contacto con profesionales de la salud ${ }^{29}$.

Existen diferentes estrategias para abordar a las personas fumadoras. Una de las más aceptadas divide las intervenciones en mínimas, breves e intensivas, de acuerdo con el tiempo que dedica el profesional a dialogar con el paciente sobre este tema ${ }^{30}$. Si bien las intervenciones intensivas suelen quedar relegadas a los especialistas, se ha sugerido que todos los profesionales que entran en contacto con personas fumadoras deberían establecer acciones concretas para alentarlos a abandonar su adicción ${ }^{30}$. Como contrapartida debe tenerse presente que la efectividad de cualquier intervención en cesación tabáquica dependerá de que sea realizada de forma apropiada. Así, dedicar un tiempo prolongado, pero de forma inapropiada puede resultar casi fútil.

Si bien idealmente el abordaje de los pacientes fumadores debería ocupar un lugar preponderante dentro del conocimiento sobre prevención cardiovascular de los residentes de cardiología, existe evidencia de que incluso pequeñas acciones pueden generar un impacto considerable. Ya en el año 1988 Ockene, et al. demostraron que programas de entrenamiento de apenas tres horas incrementaron el conocimiento sobre cesación tabáquica de residentes de medicina familiar e interna, mejorando su actitud e influencia sobre los pacientes fumadores ${ }^{12}$. Más recientemente otros estudios han hallado resultados similares después de programas de entrenamiento breves con dos sesiones de media jornada $^{15,16}$, utilizando material audiovisual, clases y juego de roles $^{17,31}$, con persistencia de estas habilidades en los residentes al ser reevaluados después de un año de su entrenamiento ${ }^{15,16}$. También han demostrado gran utilidad las rotaciones de los residentes en equipos específicos de cesación tabáquica ${ }^{32}$.

Nuestro estudio posee algunas limitaciones que merecen ser tenidas en cuenta. En primer lugar, se utilizó un muestreo no probabilístico, lo cual impide asegurar que los resultados son extrapolables al total de residentes de cada país. Sin embargo, en todos los casos la proporción de residentes que participaron en la encuesta superó la cuarta parte del total de residentes en la especialidad de cada país. Además, debe considerarse que no hay motivos para inferir que aquellos residentes que no respondieron la encuesta posean un conocimiento superior en la materia. En segundo lugar, no se dispone de los datos de filiación de los residentes ni de las características de sus centros formadores. Si bien algunas características personales como sexo o edad no deberían ser un factor que modifique su actitud frente al tabaquismo, otras cuestiones como el año de residencia o algunas características de los centros podrían ejercer cierta influencia. En tercer lugar, obtuvimos una diferente proporción de respuestas entre residentes de los distintos países respecto del total de residentes en cada sitio. Si bien esto influye en las comparaciones entre países entendemos que las similitudes en las respuestas implican una elevada homogeneidad desde el punto de vista clínico. Finalmente debe considerarse que este cuestionario no tiene la capacidad de valorar habilidades como la empatía y la escucha activa, ejes de la terapia cognitivo-conductual para la cesación tabáquica. Esto es una limitación mayor a la hora de interpretar las respuestas positivas en torno a la autopercepción de las habilidades comunicativas para lograr el abandono de la adicción. Sin embargo, consideramos que, frente a los resultados de la encuesta, no se altera la esencia de los hallazgos.

A pesar de las limitaciones enunciadas, hasta la fecha este es el primer estudio multicéntrico en valorar el grado de conocimiento y las prácticas en cesación tabáquica de cardiólogos en formación en seis países de habla hispana.

\section{Conclusión}

Si bien la mayoría de los residentes de cardiología consideran muy relevante el problema del tabaquismo y las intervenciones para su abandono, el grado de formación para afrontar esta temática es bajo. Un número muy importante de participantes expresaron no sentirse capacitados para afrontar esta tarea, y gran parte de ellos manifestaron basar sus estrategias de intervención en la derivación a otros especialistas. Un número reducido de residentes expresó emplear fármacos para lograr la cesación tabáquica de sus pacientes y, entre aquellos que los utilizaban, la mayoría estaban familiarizados con una única alternativa. Todas estas observaciones fueron similares independientemente del país de residencia de los participantes.

Consideramos crucial incluir estos tópicos dentro del programa formativo de los nuevos cardiólogos a fin de que puedan brindar una atención más integral a sus pacientes en la práctica diaria.

\section{Agradecimientos}

Los autores expresamos nuestro agradecimiento a todos los residentes que participaron desinteresadamente de este proyecto, ya que sin su valiosa colaboración no habría sido posible concretarlo. 


\section{Financiamiento}

La presente investigación no ha recibido ninguna beca específica de agencias de los sectores público, comercial o sin ánimo de lucro.

\section{Conflicto de intereses}

Los autores declaran no tener conflicto de intereses.

\section{Responsabilidades éticas}

Protección de personas y animales. Los autores declaran que para esta investigación no se han realizado experimentos en seres humanos ni en animales.

Confidencialidad de los datos. Los autores declaran que en este artículo no aparecen datos de pacientes.

Derecho a la privacidad y consentimiento informado. Los autores declaran que en este artículo no aparecen datos de pacientes.

\section{Bibliografía}

1. Barua RS, Rigotti NA, Benowitz NL, Cummings KM, Jazayeri MA, Morris PB, et al. 2018 ACC Expert Consensus Decision Pathway on Tobacco Cessation Treatment: A Report of the American College of Cardiology Task Force on Clinical Expert Consensus Documents. J Am Coll Cardiol. 2018;72(25):3332-65.

2. Jiménez-Ruiz CA, Chatkin JM, Morais A, Zabert G, Rosa P, Gea J, et al. Consensus Document on Medical Faculty Education on the Treatment of Smoking. Arch Bronconeumol. 2020 Jun 6:S0300-2896(20)30141-1 doi: 10.1016/j.arbres.2020.04.021. Online ahead of print.

3. Instituto Nacional de Estadística y Censos. $4^{\circ}$ Encuesta Nacional de Factores de Riesgo. Resultados definitivos [Internet]. Argentina: Instituto Nacional de Estadística y Censos; octubre de 2019 [consultado: 29 de junio de 2020]. Disponible en: https://www.indec.gob.ar/ftp/cuadros/publicaciones/enfr 2018 resultados definitivos.pdf

4. Ministerio de Sanidad, Consumo y Bienestar Social. Encuesta Nacional de Salud de España 2017 [Internet]. España: Ministerio de Sanidad Consumo y Bienestar Social; 26 de junio de 2018 [consultado: 29 de junio de 2020]. Disponible en: https://www.mscbs.gob.es/estadEstudios/estadisticas/encuestaNacional/encuestaNac2017/ENSE17_pres web.pdf

5. Ministerio de Salud. Encuesta Nacional de Salud 2016-2017. Primeros resultados [Internet]. Chile: Ministerio de Salud, Departamento de Epidemiología, División de Planificación Sanitaria, Subsecretaría de Salud Pública [consultado: 29 de de junio de io de 2020]. Disponible en: https:// www.minsal.cl/wp-content/uploads/2017/11/ENS-2016-17_PRIMEROS-RESULTADOS.pdf

6. Bardach A, Cañete F, Sequera VG, Palacios A, Alcaraz Am, Rodríguez B et al. Carga de enfermedad atribuible al uso del tabaco en Paraguay y potencial impacto sanitario y económico del aumento del precio a través de impuestos. Rev Peru Med Exp Salud Publica. 2018;35(4):599-609.

7. Instituto Nacional de Estadística de Uruguay. Encuesta mundial de tabaquismo en adultos. Uruguay 2017 [Internet]. Uruguay: Instituto Nacional de Estadística [consultado: 29 de junio de 2020]. Disponible en: http:// ine.gub.uy/c/document library/get file?uuid=09a14fa0-9cbf-40bb-a55a-a $29892240 \mathrm{~d} 7 \mathrm{a} \&$ groupld $=10181$

8. Comisión Nacional contra las Adicciones. Instituto Nacional de Salud Pública. Encuesta Global de Tabaquismo en Adultos México 2015 [Internet]. México: Secretaría de Salud [consultado 29 de junio de 2020]. Disponible en: http://omextad.salud.gob.mx/contenidos/encuestas/ gats2015/ENCUESTA_GATS_2015.pdf

9. Ralston J. World Heart Federation and the UN High-Level Meeting on Non-Communicable Diseases (NCDs): Advocacy and action to address the global burden of cardiovascular and other NCDs. Global Heart. 2011;6(3):65-6.
10. Kontis V, Mathers CD, Rehm J, Stevens GA, Shield KD, Bonita R, et al. Contribution of six risk factors to achieving the $25 \times 25$ non-communicable disease mortality reduction target: a modelling study. Lancet. 2014; 384(9941):427-37.

11. Bickerstaffe G. Smoking cessation for hospital inpatients. BMJ Qual Improv Rep. 2014;3(1):u204964.w2110.

12. Ockene JK, Quirk ME, Goldberg RJ, Kristeller JL, Donnelly G, Kalan KL, et al. A residents' training program for the development of smoking intervention skills. Arch Intern Med. 1988;148(5):1039-45.

13. Leone FT, Zhang Y, Evers-Casey S, Evins AE, Eakin MN, Fathi J, et al. Initiating pharmacologic treatment in tobacco-dependent adults. An Official American Thoracic Society Clinical Practice Guideline. Am J Respir Crit Care Med. 2020;202(2):e5-e31.

14. López-Sendón J, González-Juanatey JR, Pinto F, Cuenca Castillo J, Badimón L, Dalmau R, et al. Quality markers in cardiology. Main markers to measure quality of results (outcomes) and quality measures related to better results in clinical practice (performance metrics). INCARDIO (Indicadores de Calidad en Unidades Asistenciales del Area del Corazón): A SEC/SECTCV Consensus Position Paper. Rev Esp Cardiol. 2015; 68(11):976-95.e10.

15. Cornuz J, Humair JP Seematter L, Stoianov $R$, van Melle G, Stalder $H$ et al. Efficacy of resident training in smoking cessation: a randomized controlled trial of a program based on application of behavioral theory and practice with standardized patients. Ann Intern Med. 2002;136(6):429-37.

16. Humair JP, Cornuz J. A new curriculum using active learning methods and standardized patients to train residents in smoking cessation. J Gen Intern Med. 2003;18(12):1023-7.

17. Steinemann S, Roytman T, Chang J, Holzman J, Hishinuma E, Nagoshi M, et al. Impact of education on smoking cessation counseling by surgical residents. Am J Surg. 2005;189(1):44-6.

18. Pack QR, Keteyian SJ, McBride PE, Weaver WD, Kim HE. Current status of preventive cardiology training among United States cardiology fellowships and comparison to training guidelines. Am J Cardiol. 2012;110(1):124-8.

19. Landis ZC, Rolius R, Scott IU. Practice patterns among eye care providers at US teaching hospitals with regard to assessing and educating patients about smoking. Am J Ophthalmol. 2017;180:86-90.

20. Nims L, Jordan TR, Price JH, Dake JA, Khubchandani J. Smoking cessation education and training in obstetrics and gynecology residency programs in the United States. J Family Med Prim Care. 2019;8(3):1151-8.

21. GBD 2015 Tobacco Collaborators. Smoking prevalence and attributable disease burden in 195 countries and territories, 1990-2015: a systematic analysis from the Global Burden of Disease Study 2015. Lancet. 2017;389(10082):1885-906.

22. Fernández de Bobadilla J, Dalmau R, Galve E; Legislation on Tobacco and Acute Coronary Syndrome in Spain Group. Laws restricting smoking in public places reduce the incidence of acute coronary syndrome in Spain. Rev Esp Cardiol. 2014;67(5):349-52.

23. van den Berg MJ, van der Graaf Y, Deckers JW, de Kanter W, Algra A Kappelle LJ, et al. Smoking cessation and risk of recurrent cardiovascular events and mortality after a first manifestation of arterial disease. Am Heart J. 2019;213:112-22.

24. Riley H, Ainani N, Turk A, Headley S, Szalai H, Stefan M, et al. Smoking cessation after hospitalization for myocardial infarction or cardiac surgery: Assessing patient interest, confidence, and physician prescribing practices. Clin Cardiol. 2019;42(12):1189-94.

25. Aziz O, Skapinakis $P$, Rahman S, Rao C, Ashrafian H, Panesar SS, et al. Behavioural interventions for smoking cessation in patients hospitalised for a major cardiovascular event. Int J Cardiol. 2009;137(2):171-4.

26. Feeney GF, McPherson A, Connor JP, McAlister A, Young MR, Garrahy P. Randomized controlled trial of two cigarette quit programmed in coronary care patients after acute myocardial infarction. Intern Med J. 2001;31(8):470-5.

27. Munafò M, Rigotti N, Lancaster T, Stead L, Murphy M. Interventions fo smoking cessation in hospitalized patients: a systematic review. Thorax. 2001;56(8):656-63

28. Rigotti NA, Clair C, Munafò MR, Stead LF. Interventions for smoking cessation in hospitalized patients. Cochrane Database Syst Rev. 2012;(5):CD001837

29. Mellon L, McElvaney NG, Cormican L, Hickey A, Conroy R, Ekpotu L, et al. Determining rates of smoking cessation advice delivered during hospitalisation and smoking cessation rates 3 months post discharge: a two-hospital survey. Health Psychol Behav Med. 2016;4(1):124-37.

30. 2008 PHS Guideline Update Panel, Liaisons, and Staff. Treating tobacco use and dependence: 2008 update U.S. Public Health Service Clinical Practice Guideline executive summary. Respir Care. 2008;53(9):1217-222.

31. Movsisyan NK, Petrosyan V, Abelyan G, Sochor O, Baghdasaryan S, Etter JF. Learning to assist smokers through encounters with standardized patients: An innovative training for physicians in an Eastern European country. PLoS One. 2019;14(9):e0222813

32. O'Sullivan MM, Hoskote SS, Lesko MB, Mallozzi CM, Lee YI, Fayanju OA et al. Rotation in a smoking cessation clinic improves nicotine dependence treatment provided by first-year internal medicine trainees. Fam Med. 2016;48(6):472-6. 